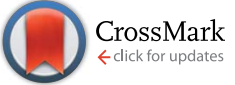

Cite this: RSC Adv., 2017, 7, 14481
Received 20th December 2016 Accepted 28th February 2017

DOI: 10.1039/c6ra28482e

rsc.li/rsc-advances

\section{Realization of uniaxially strained, rolled-up monolayer CVD graphene on a Si platform via heteroepitaxial InGaAs/GaAs bilayers}

\author{
Guoming Mao, Qi Wang, ${ }^{*}$ Zhaoer Chai, Hao Liu, Kai Liu and Xiaomin Ren \\ III-V semiconductor/graphene tubular structures with diameters of 4.5-5.4 $\mu \mathrm{m}$ have been fabricated on \\ a silicon platform by rolling up monolayer CVD graphene together with heteroepitaxial InGaAs/GaAs \\ bilayers. Scanning electron microscopy (SEM) reveals that transferred graphene adheres to the wall of \\ the Si-based InGaAs/GaAs microtube. Micro-Raman spectroscopy measurements show remarkable \\ redshifts of the $G$ and $2 D$ bands of graphene after planar graphene totally rolls up, reflecting that rolled- \\ up graphene is under uniaxial tensile strain and the strain originates from the rolled-up InGaAs/GaAs \\ microtube. We also fabricated GaAs-based III-V semiconductor/graphene tubular structures with \\ diameters of 3.7 and $4.7 \mu \mathrm{m}$, respectively, thus finding an approach to graphene strain engineering (i.e., \\ the Raman redshift and tensile strain of rolled-up graphene increase with the decrement of microtube \\ diameter). Obviously, assembling strained graphene with III-V semiconductors in rolled-up form on a Si \\ platform will bring about a variety of Si-based electronic and optical applications in the future.
}

\section{Introduction}

Graphene, a two-dimensional (2D) form of $\mathrm{sp}^{2}$-bonded carbon atoms, has sparked great attention and tremendous research interest since it was discovered in $2004 .{ }^{1}$ Due to the fact that graphene possesses unique mechanical, ${ }^{2}$ electrical, ${ }^{3}$ optical, ${ }^{4}$ magnetic ${ }^{5}$ and thermal ${ }^{6}$ properties, a variety of exciting applications of graphene are being vigorously explored by academia and industry. Particularly, the intrinsic properties of graphene can be tuned by heteroatom doping, ${ }^{7}$ zigzag nanoribbons, ${ }^{8}$ applied strain, ${ }^{9}$ heterojunctions, ${ }^{10}$ and nanocomposites, ${ }^{11}$ which is very important for exploiting new phenomena for advanced graphene applications.

In recent years, the integration and interaction of graphene with conventional semiconductors, especially GaAs, has aroused great interest. 18.5\% efficient graphene/GaAs van der Waals heterostructure solar cells, ${ }^{12}$ a transmission filter based on stacked graphene/GaAs/graphene layers operating at the $1.55 \mathrm{~mm}$ wavelength ${ }^{13}$ and other applications have already investigated. Meanwhile, different kinds of interactions between graphene and GaAs have been studied, including electron-hole pairing in graphene/ GaAs heterostructures, ${ }^{14}$ the phonon assisted cyclotron resonance effect in single-layer graphene on GaAs ${ }^{15}$ piezoelectric surface acoustical phonon amplification in graphene on GaAs, ${ }^{16}$ magneto-transport properties of exfoliated graphene on GaAs. ${ }^{17}$ Related potential devices utilizing these interactions, such as

State Key Laboratory of Information Photonics and Optical Communications, Beijing University of Posts and Telecommunications, Beijing 100876, China. E-mail: wangqi@bupt.edu.cn analog to digital converters, topologically protected quantum bits, acoustical phonon amplifier and frequency tunable acoustical phonon device, were also imagined. All these research studies show us the tremendous advantages of integrating strained graphene with conventional semiconductors.

On the other hand, as a type of building block for 3D micro- $/$ nano-architectures, self-rolled-up tubes also stimulate significant interest of researchers. Generally, two or more stacked strained layers are grown on the top of a sacrificial layer (SCL), followed by releasing from substrate and forming tubular structure through the selective etching of SCL. Self-rolled-up tubes have naturally hollow channel as well as good controllability of their diameter, length and wall. A variety of applications including electrically-injected tube laser, ${ }^{18}$ field effect transistors, ${ }^{19}$ fluidic sensing ${ }^{20}$ and lithium ion batteries (LIBs), ${ }^{21}$ have been envisioned and realized by self-rolled-up tubes.

Recently, physically combining graphene with rolled-up tubes, i.e., producing homogenously curved graphene structures through a nonlocal bending method, has been actively pursued. Several kinds of tubular hybrid graphene heterostructures have been reported. For example, Chen et al. reported strain-driven formation of multilayer $\mathrm{rGO} / \mathrm{GeO}_{2}$ microtubes, which can serve as durable anodes with satisfactory cycling ability and high capacity for lithium ion batteries. ${ }^{22}$ Liu et al. designed the sandwich nanoarchitecture of rolled-up Si/rGO bilayer nanomembranes, improving the cycling performance of silicon anodes of LIBs through rGO conductive protecting layers. ${ }^{21}$ In particular, Barcelos et al. produced rolled-up InGaAs/ $\mathrm{Cr} / \mathrm{Graphene}$ microtube on GaAs substrates and indicated that rolled-up graphene is free of strain, which implies interaction of 
graphene with additional materials on the heterostructure is very weak. ${ }^{23}$ Obviously, such combing approach has the advantage of keeping or tuning the tubular graphene heterojunctions in a well-controlled fashion. However, there still remain some underlying drawbacks in terms of future applications. First, use of rGO in rolled-up nanomembranes can hardly avoid introducing defects, which is unfavorable for some rolled-up graphene devices. Secondly, opening and tuning bandgap of as-obtained strain-free CVD-grown graphene through applying uniaxial stress is almost impossible.

In this study, III-V semiconductor/CVD-grown-graphene tubular structure arrays have been integrated on silicon platform. Scanning electron microscope (SEM) clearly shows the attachment between III-V semiconductor and graphene. More inspiringly, significant Raman redshifts of $\mathrm{G}$ and $2 \mathrm{D}$ bands are observed from rolled-up graphene. Through analysis, we conclude that rolled-up graphene is under uniaxial tensile strain, which originates from the strain relaxation during selfrolling process. After that, we fabricated GaAs based III-V semiconductor/graphene tubular structures with typical diameters of $3.7 \mu \mathrm{m}$ and $4.7 \mu \mathrm{m}$ and a regulation of the strain state of graphene by the tube diameter has been realized (uniaxially tensile strain of rolled-up graphene increase with the decrement of microtube diameter). Above results also prove the formation of III-V semiconductor/graphene heterojunctions. As far as we know, it is the first time to regulate strained graphene through the rolled-up forms. As-fabricated III-V semiconductor/ strained-graphene tubular structures have great potential to be used in sensors, capacitors, microfluidics devices and etc. Most importantly, this study opens a smart path to combine III$\mathrm{V}$ semiconductor with graphene on traditional Si platform leveraging the mature Si CMOS technology and low-cost largearea substrates, and undoubtedly bringing impact to Si-based electronics and photonics.

\section{Experimental details}

Si-based metamorphic InGaAs/GaAs heterostructures for rolling up microtubes were grown by low-pressure metal-organic chemical vapor deposition (LP-MOCVD). The layer stacks on Si (100) substrate consist of a $2 \mu \mathrm{m}$-thick GaAs buffer and a 50 nm-thick AlAs SCL, followed by a $15 \mathrm{~nm}$-thick $\mathrm{In}_{0.2} \mathrm{Ga}_{0.8} \mathrm{As}$ compressive-strained layer and a $35 \mathrm{~nm}$-thick GaAs layer as top bilayer. The growth details can be found in our previous work. ${ }^{24}$ Periodic rectangular patterns (lengths ranging from $50 \mu \mathrm{m}$ to $250 \mu \mathrm{m}$, widths ranging from $30 \mu \mathrm{m}$ to $70 \mu \mathrm{m}$ ), separated by 50 $\mu \mathrm{m}$, were defined on the wafer along the [001] direction by first photolithography. After that, a shallow etch to AlAs sacrificial layer by $\mathrm{H}_{2} \mathrm{SO}_{4}-\mathrm{H}_{2} \mathrm{O}_{2}-\mathrm{H}_{2} \mathrm{O}(1: 1: 60)$ solution transferred the patterns into InGaAs/GaAs bilayers and formed the mesas (Fig. 1a). As shown in Fig. 1b, CVD-grown monolayer graphene flake with size of $1 \mathrm{~cm} \times 1 \mathrm{~cm}$ was then transferred to the top of wafer, the transfer details are described below. Subsequently, a secondary photolithography was performed to produce stripes with $80 \mu \mathrm{m}$ in width, followed by oxygen $\left(\mathrm{O}_{2}\right)$ plasma etching of graphene, which exposes part of the mesas and provides the etching front (Fig. 1c and e). Finally, highly selective $\mathrm{HF}-\mathrm{H}_{2} \mathrm{O}$

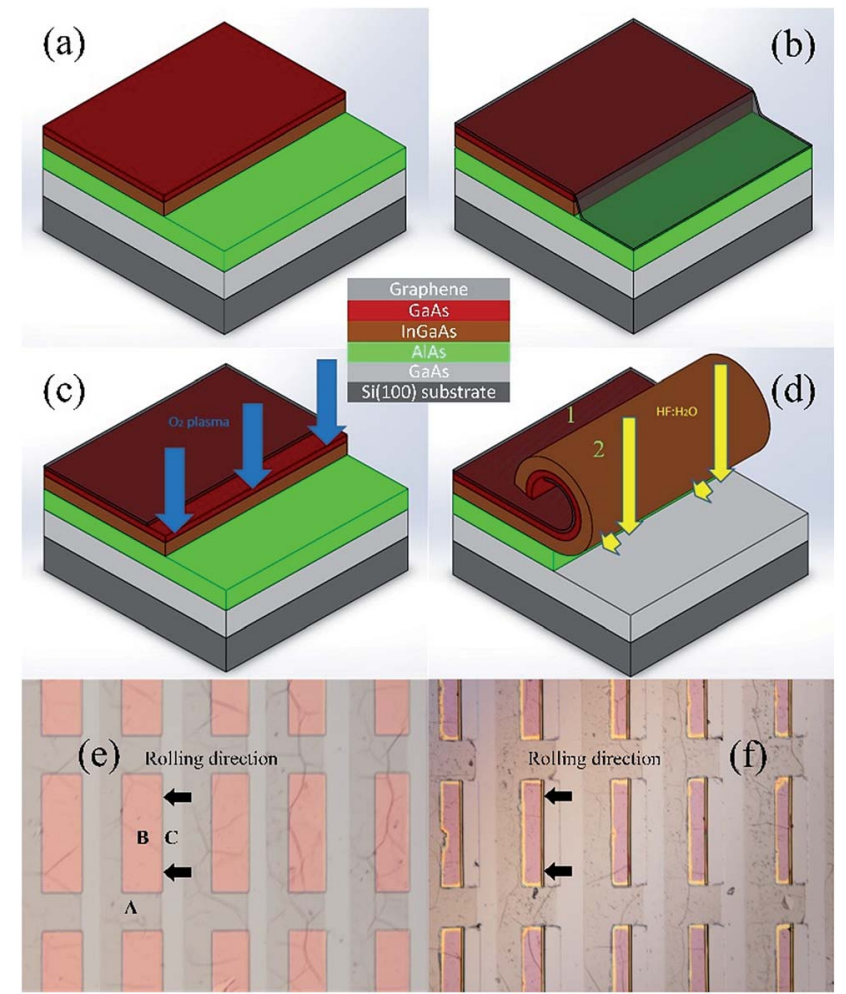

Fig. 1 Process flows for InGaAs/GaAs/graphene tubular structures. (a-d) Schematic illustrations of production steps: (a) shallow wet etching into AlAs forms the rectangular mesas. (b) Transferring CVD graphene onto the mesas realizes total coverage. (c) Dry etching of graphene by $\mathrm{O}_{2}$ plasma exposes the sidewall of the mesa. (d) Lateral selective etching of AlAs SCL releases the layer stacks into tubular forms. Inset shows schematic diagram of the layer stacks. Number 1 and 2 in (d) represent the chosen measurement points for microRaman. (e and f) Optical microscopy images of unrolled planar and rolled-up InGaAs/GaAs/graphene tubes. Dark arrows indicate the rolling direction. Region A (translucent, wrinkled region), B (pink color) and $C$ (grey color) represent graphene, graphene-capped InGaAs/ GaAs bilayers and exposed AlAs, respectively.

(1:40) dilute solution started to laterally remove AlAs SCL, which rolled up the graphene with InGaAs/GaAs bilayer from the [010] direction due to strain relaxation (Fig. 1d and f). 15/ $35 \mathrm{~nm}$ and $10 / 20 \mathrm{~nm} \mathrm{In}_{0.2} \mathrm{Ga}_{0.8} \mathrm{As} / \mathrm{GaAs}$ bilayers grown on GaAs (100) substrate were also used to roll up graphene in this study.

The monolayer graphene used in this study was synthesized on copper foils by chemical vapor deposition (CVD). A supporting layer of polymethyl methacrylate (PMMA) was then spin-coated onto the surface of graphene. Copper substrate was finally etched away from $\mathrm{Cu} / \mathrm{graphene/PMMA}$ foils with $\mathrm{Cu}$ etchant, obtaining delicate graphene/PMMA stacks, followed by thorough rinsing in deionized water. After graphene/PMMA was transferred to GaAs/Si virtual substrate with minimized folding or tearing of graphene layer in DI water, pre-coated PMMA supporting layer was dissolved in acetone..$^{25,26}$

Optical microscope observation was used to evaluate photolithography quality and monitor the transfer process of CVD graphene. Scanning electron microscopy (SEM) measurements were carried out to characterize the morphological and 
structural properties of the III-V semiconductor/graphene tubular structures. In order to investigate the optical properties of graphene and III-V semiconductors, room temperature (RT) micro-Raman spectra were collected using a Renishaw inVia confocal Raman spectrometer in back scattering geometry. The excitation wavelength was fixed to $532 \mathrm{~nm}$ and the laser power was $2 \mathrm{~mW}$. Laser beam was focused on the sample by a $100 \times$ objective lens (numerical aperture $=0.85$ ), resulting in a spot size of $\sim 3 \mu \mathrm{m}$ in diameter.

\section{Results and discussion}

SEM visualization of highly-ordered InGaAs/GaAs/graphene tube arrays on Si platform is displayed in Fig. 2a. We note that as-fabricated tubes are quite homogeneous along the entire length, proving the perfect self-rolling process. Fig. $2 \mathrm{~b}$ shows that a single InGaAs/GaAs/graphene tube with total length of $140 \mu \mathrm{m}$ rolls over the distance of $\sim 20 \mu \mathrm{m}$, which therefore determines the number of rotations according to the measured tube diameter. Simultaneously, planar areas are divided into different parts and then labelled in Fig. $2 \mathrm{~b}$ according to their background colours. In addition, very smooth and crackless tube wall can be clearly observed in Fig. 2b. As shown in Fig. 2c, direct SEM view of rolled-up monolayer CVD graphene inside microtube was realized from the deliberately-broken position, clearly showing that monolayer graphene adheres to the inner wall of Si-based InGaAs/GaAs microtube, thus forming III-V semiconductor/graphene tubular heterojunction directly on silicon substrate. Fig. $2 \mathrm{c}$ also shows that the outer diameter of

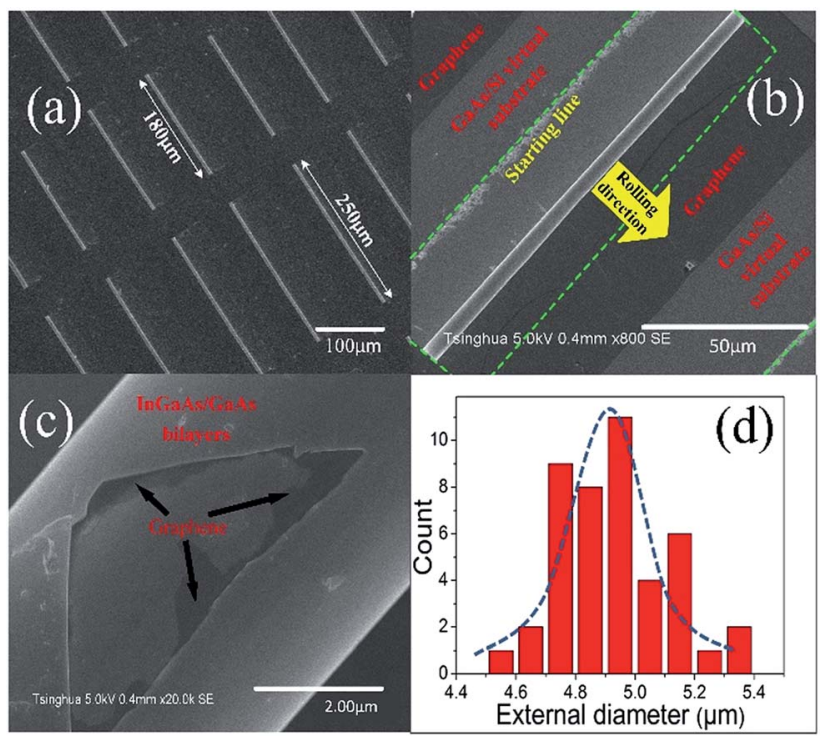

Fig. 2 (a) SEM image of highly-ordered arrays of rolled-up InGaAs/ GaAs/graphene microtubes with two typical lengths of 180 and 250 $\mu \mathrm{m}$. (b) SEM image of a single InGaAs/GaAs/graphene tube with length of $140 \mu \mathrm{m}$. The broken green lines show the border of rectangular mesas. (c) Magnified SEM image of deliberately-broken part of a single microtube with the outer diameter of $5 \mu \mathrm{m}$. Transferred graphene was exposed and III-V semiconductor/graphene tubular heterojunctions can be clearly distinguished. (d) Histogram of the outer diameter of asfabricated InGaAs/GaAs/graphene tubes measured by SEM.
InGaAs/GaAs/graphene microtube is $\sim 5 \mu \mathrm{m}$ and the tube performs more than 1 rotation (two InGaAs/GaAs bilayer stacks can be seen). In addition, the windings of InGaAs/GaAs/ graphene microtube are quite tight (i.e., neighbouring bilayers are perfectly joined together), also demonstrating high quality of the rolling process. To further assess the quality of microtube arrays, we measured more than 40 tubes from the array by SEM and summarized the statistical results of the diameter into a histogram. As shown in Fig. 2e, diameter distribution of the tubes concentrating at about $4.9 \mu \mathrm{m}$ can be observed, indicating as-prepared microtube array is quite uniform. Diameter deviation can be interpreted by a slightly disturbed roll-up process.

Subsequently, we measured micro-Raman spectra from nonrolled-up and rolled-up parts (i.e., point 1 and 2 in Fig. 1d) of a single Si-based InGaAs/GaAs/graphene microtube with diameter of $\sim 5 \mu \mathrm{m}$. As appearing very far away from that of III-V semiconductors (e.g., GaAs, AlAs and InGaAs), Raman peaks of graphene can be easily distinguished from the full spectra. In order to clearly show their discrepancy, normalized Raman spectra of III-V semiconductors and graphene are individually shown in Fig. 3 and 4. Fig. 3 highlights the Raman spectra of Si-based rolled-up InGaAs/GaAs bilayer and its corresponding planar (with graphene), in which the longitudinal-optical (LO) phonon modes of top GaAs layer are identified as the strongest peaks around 287$292 \mathrm{~cm}^{-1}$. LO phonon mode of AlAs SCL contributes to the small peak ranging at $\sim 399 \mathrm{~cm}^{-1}$ in the spectrum of planar InGaAs/ GaAs bilayer, which vanishes in the spectrum of rolled-up microtubes due to the complete etching off of AlAs after tube formation. Moreover, the broad and weak spectral features in the range of 260-270 $\mathrm{cm}^{-1}$ originate from the signals of transverse optical (TO) phonon modes of top GaAs layer. When the InGaAs/GaAs strained bilayer rolls up into microtube together with CVD graphene, -2 $\mathrm{cm}^{-1}$ redshift $\left(\Delta \omega \sim-2 \mathrm{~cm}^{-1}\right.$, from 289.3 to $287.3 \mathrm{~cm}^{-1}$ ) and the increment of peak full width at half maximum (FWHM) of LO GaAs peak are simultaneously observed. The spectral redshift and broadening of LO GaAs Raman peak should be induced by the strain state transition of the top GaAs layer during rolling process.

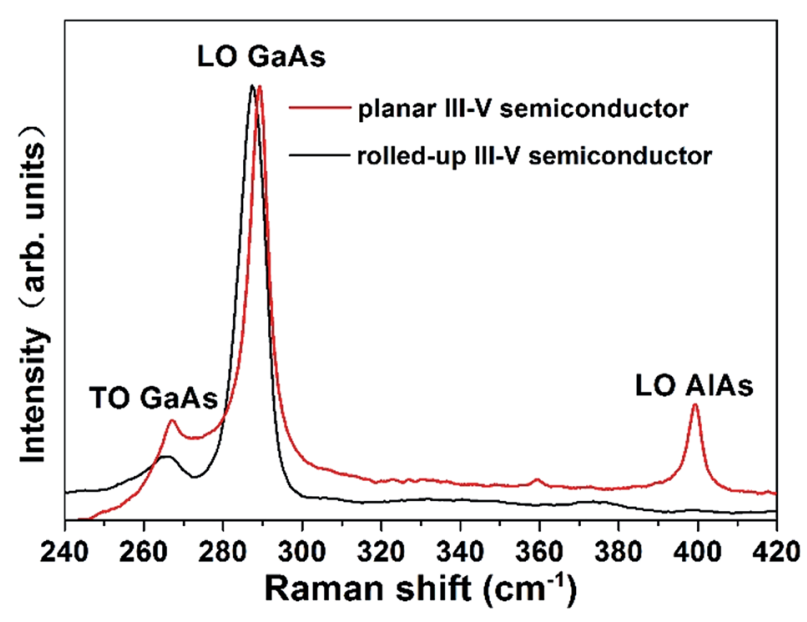

Fig. 3 Raman spectra of Si-based rolled-up InGaAs/GaAs bilayer and corresponding planar (with graphene on surface). TO GaAs, LO GaAs, and LO AlAs peaks are marked, respectively. 
(a)

Raman spectra of planar (non-rolled-up) and rolled-up graphene are simultaneously illustrated in Fig. 4a, from which sharp $\mathrm{G}$ and 2D bands as well as very weak D bands (defectrelated) originating from monolayer CVD graphene flake are clearly visible. After the planar graphene rolls up into microtube, Raman intensity of graphene $\mathrm{D}$ band is slightly increased and meanwhile $2 \mathrm{D} / \mathrm{G}$ intensity ratio $\left(I_{2 \mathrm{D}} / I_{\mathrm{G}}\right)$ is slightly decreased from 3.2 to 2.5 , from which we can identify that high-quality self-rolling process only introduces very few defects into graphene. Fig. $4 \mathrm{~b}$ and $\mathrm{c}$ are the magnified parts of $\mathrm{G}$ and $2 \mathrm{D}$ bands of Fig. 4a, which clearly reveal the Raman shift of $G$ and $2 \mathrm{D}$ bands after planar graphene totally rolled up. Surprisingly, average redshifts of $\mathrm{G}$ and $2 \mathrm{D}$ bands of graphene were up to $-8.1 \mathrm{~cm}^{-1}\left(\Delta_{\mathrm{G}}\right.$, from 1585.2 to $\left.1577.1 \mathrm{~cm}^{-1}\right)$ and $-26.6 \mathrm{~cm}^{-1}$ ( $\Delta_{2 \mathrm{D}}$, from 2679.8 to $2653.2 \mathrm{~cm}^{-1}$ ), respectively. In addition, peak FWHM of G band increases from 18.5 to $29.2 \mathrm{~cm}^{-1}$, that of 2D band from 36.8 to $44.9 \mathrm{~cm}^{-1}$. Mohiuddin et al. have reported that $\mathrm{G}$ band of graphene was splitted into two subbands, (i.e., G+ and G- bands) when they applied uniaxial strain to graphene monolayer, and the splitting between $\mathrm{G}+$ and $\mathrm{G}-$ bands increases with the ramp up in strain. ${ }^{27}$ In our study, asymmetric $\mathrm{G}$ band of rolled-up graphene rather than obvious splitting of $\mathrm{G}$ band is observed (see black curve in Fig. 4b). In particular, the wavenumber redshift ratio $\left(\Delta_{2 \mathrm{D}} / \Delta_{\mathrm{G}}\right)$ is calculated to be $\sim 3.3$ after planar graphene rolled-up, which just falls in the range between $\Delta_{2 \mathrm{D}} / \Delta_{\mathrm{G}^{+}}$of $\sim 5.9$ and $\Delta_{2 \mathrm{D}} / \Delta_{\mathrm{G}-}$ of $\sim 2.0$ derived by Mohiuddin's results. Moreover, the broadening of FWHM along with strain was also observed by Mohiuddin. Considering that rolled-up and planar graphene are formed in the same process flow and corresponding Raman spectroscopy for graphene are probed under the identical conditions, we thus believe that rolled-up graphene is also under tensile strain which accounts for the redshift and the broadening of $\mathrm{G}$ and $2 \mathrm{D}$ bands of graphene in this study. Finally, the experimental phenomena that there is no observation of Raman G-band splitting may be attributed to insufficient tensile strain loaded to graphene by 15/35 nm InGaAs/GaAs bilayer.

As the Raman shift of $2 \mathrm{D}$ band dominates over that of $\mathrm{G}$ band after planar graphene is rolled-up, we have further measured 2D bands of graphene from more than $40 \mathrm{Si}$-based InGaAs/GaAs/graphene microtubes and summarized the peak wavenumber of $2 \mathrm{D}$ bands into a histogram (Fig. $5 \mathrm{c}$ ), by which we have analyzed the strain state of the rolled-up graphene in detail. As shown in Fig. 5c, a bimodal Gaussian distribution of $2 \mathrm{D}$ band peaks of rolled-up graphene can be distinctly observed. Due to the fact that rolled-up graphene contacts with GaAs via van der Waals force and has monomodal diameter distribution (refer to Fig. 2d), we think this bimodal Gaussian distribution can be well explained by two types of typical contact intensity between rolled-up graphene and top GaAs layer. We used two Gaussian curves to fit the envelope of the histogram in Fig. $5 \mathrm{c}$ and got two peaks. Left peak around $2652 \mathrm{~cm}^{-1}$, named as $\mathrm{GP}_{1}$, manifests that rolled-up graphene perfectly contacts with InGaAs/GaAs bilayer and thus sustains all the tensile strain
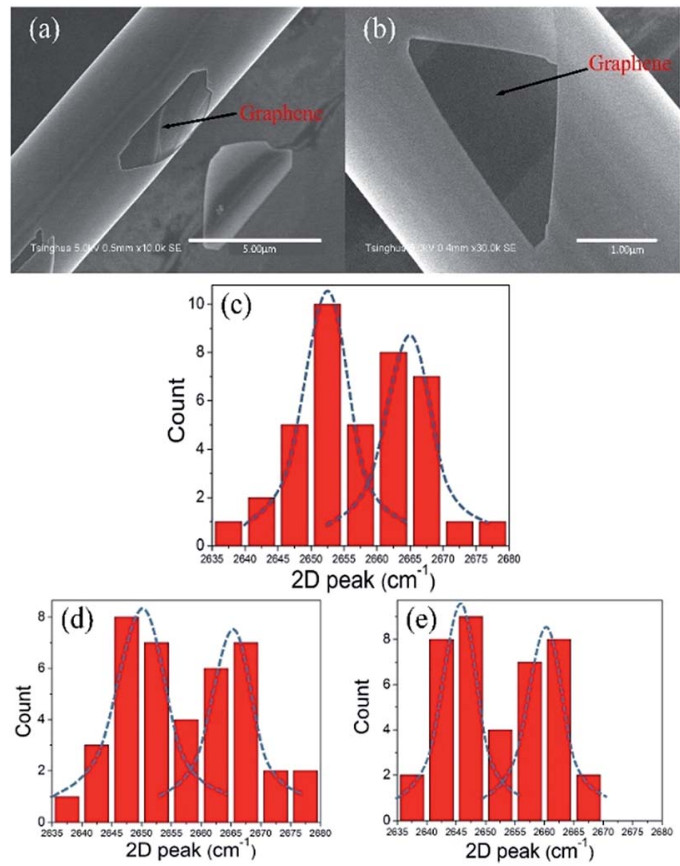

Fig. 5 (a and b) SEM images of GaAs-based InGaAs/GaAs/graphene microtubes fabricated by $15 / 35 \mathrm{~nm}$ (a) and $10 / 20 \mathrm{~nm}$ (b) strained bilayers, respectively. The outer diameter of microtube is $4.7 \mu \mathrm{m}$ (a) and $3.7 \mu \mathrm{m}$ (b), respectively. Transferred CVD graphene was exposed by deliberately breaking InGaAs/GaAs bilayers of the microtubes and graphene wrinkle can be observed. (c-e) Histogram of peak wavenumber of graphene 2D bands which originate from Si-based (15/35 $\mathrm{nm}$ ) and GaAs-based InGaAs/GaAs/graphene microtubes (15/35 nm, 10/20 $\mathrm{nm})$, respectively. 
loaded by rolled-up GaAs. While, the other co-existing Gaussian peak $\left(\mathrm{GP}_{2}\right)$, situating around $2665 \mathrm{~cm}^{-1}$, suggests that rolled-up graphene with GaAs is not very tight due to the surface wrinkles of graphene.

We also fabricated GaAs-based InGaAs/GaAs/graphene microtubes using $15 / 35 \mathrm{~nm}$ and $10 / 20 \mathrm{~nm} \mathrm{In}_{0.2} \mathrm{Ga}_{0.8} \mathrm{As} / \mathrm{GaAs}$ strained bilayers, respectively. Typical diameters of $4.7 \mu \mathrm{m}$ and $3.7 \mu \mathrm{m}$ can be calculated from SEM images shown in Fig. $5 \mathrm{a}$ and b. Moreover, we deliberately broke the outer wall of the GaAs-based microtube, enabling rolled-up CVD graphene flake exposed and easily distinguished from InGaAs/GaAs bilayer. We summarized Raman peak distribution of graphene 2D band measured from several tens of GaAs-based tubes. As shown in Fig. 5d and e, bimodal Gaussian distribution of 2D band peaks of rolled-up graphene has been observed again. Both $\mathrm{GP}_{1}$ and $\mathrm{GP}_{2}$ peaks of the rolled-up graphene in microtube with diameter of $3.7 \mu \mathrm{m}$ redshifted $\sim 5 \mathrm{~cm}^{-1}$ compared with the corresponding peaks in microtube with diameter of $4.7 \mu \mathrm{m}$, reflecting that uniaxial strain of roll-up graphene is increased with the increase of microtube curvature. Obviously, the dependence of the band shift of rolled-up graphene on the diameter should be valid in Si-based InGaAs/GaAs/graphene microtubes. Subsequently, we carefully examine Fig. $5 \mathrm{c}$ and $\mathrm{d}$ and then find that $\mathrm{GP}_{1}$ of Sibased tubes fabricated by $15 / 35 \mathrm{~nm}$ InGaAs/GaAs metamorphic bilayer was slightly blueshifted when compared with that of GaAs-based tubes using the same bilayer, while $\mathrm{GP}_{2}$ almost unchanged. This phenomenon can be interpreted from the point view of surface roughness of inner wall of microtube. Due to the intrinsic limitations in GaAs/Si metamorphic growth, GaAs-based InGaAs/GaAs bilayer has much smoother top GaAs surface than its Si-based counterpart. As $\mathrm{GP}_{1}$ peak originates from the tight attachment of rolled-up graphene onto the top GaAs, higher surface roughness of GaAs has significant effect on the $\mathrm{GP}_{1}$ peak, i.e., accommodating parts of strain energy and then making the strain decrease of rolled-up graphene. For $\mathrm{GP}_{2}$, the influence of surface roughness of top GaAs will be highly overridden by surface wrinkle of rolled-up graphene.

\section{Conclusions}

In summary, we have realized 3D III-V semiconductor/strainedgraphene tubular nanostructures on Si platform through simply rolling CVD graphene with InGaAs/GaAs bilayers. Tubular heterojunctions which are formed by the attachment of graphene and III-V semiconductor have been demonstrated by the direct SEM visualization. By analyzing micro-Raman spectra of graphene and GaAs, we can prove that uniaxial tensile strain is applied onto rolled-up monolayer CVD graphene and the strain value of rolled-up graphene can be regulated by the diameter of InGaAs/GaAs/graphene microtube. Importantly, as graphene is easily functionalized and microtube can provide a native microfluidic channel, obtained III-V semiconductor/graphene tubular structure has great potential of applications in biosensors, gas sensors, MEMS/NEMS, lab-in-a-tube system and etc. Meanwhile, graphene is located inside of the microtube rather than directly exposed to the atmosphere, which can improve the long-term stability of some applications based on tubular heterojunctions.
Finally, integration of strained graphene with traditional semiconductors on silicon platform in such an easy way will make some envisaged electronics and photonics applications true.

\section{Acknowledgements}

This work was supported by the National Natural Science Foundation of China (No. 61474008), Program for New Century Excellent Talents in University (NCET-13-0686), International Science \& Technology Cooperation Program of China (No. 2011DFR11010) and the 111 Project (No. B07005).

\section{References}

1 K. S. Novoselov, A. K. Geim, S. V. Morozov, D. Jiang, Y. Zhang, S. V. Dubonos, I. V. Grigorieva and A. A. Firsov, Science, 2004, 306, 666.

2 J. H. Chen, C. Jang, S. Xiao, M. Ishigami and M. S. Fuhrer, Nat. Nanotechnol., 2008, 3, 206.

3 W. H. Park, I. Jo, B. H. Hong and H. Cheong, Nanoscale, 2016, 8, 9822.

4 R. Podila, B. Anand, J. T. Spear, P. Puneet, R. Philip, S. S. S. Sai and A. M. Rao, Nanoscale, 2012, 4, 1770.

5 J. J. Chen, H. C. Wu, D. P. Yu and Z. M. Liao, Nanoscale, 2014, 6, 8814.

6 A. A. Balandin, S. Ghosh, W. Bao, I. Calizo, D. Teweldebrhan, F. Miao and C. N. Lau, Nano Lett., 2008, 8, 902.

7 B. Xie, Y. Chen, M. Yu, X. Shen, H. Lei, T. Xie, Y. Zhang and Y. Wu, Nanoscale Res. Lett., 2015, 10, 1.

8 K. P. Dou, X. X. Fu, S. A. De and R. Q. Zhang, Nanoscale, 2015, 7, 20003.

9 T. V. Amelsvoort, A. Denayer, J. Boermans and A. Swillen, Physics, 2016, 236, 206.

10 L. Wang, J. Zhao, Z. Zhang, B. Ding and Y. Guo, J. Electron. Mater., 2016, 45, 4484.

11 H. E. Heba, M. A. Shaimaa, A. N. Amr, Z. Fouad, A. E. Saad, Y. Dena, G. M. Husseinb and M. S. Atreesb, J. Hazard. Mater., 2017, 322, 370.

12 X. Li, W. Chen, S. Zhang, Z. Wu and P. Wang, Nano Energy, 2015, 16, 310.

13 H. Ajlani, M. K. Azizi, A. Gharsallah and M. Oueslati, Opt. Mater., 2016, 57, 120.

14 A. Gamucci, D. Spirito, M. Carrega, B. Karmakar and A. Lombardo, Nat. Commun., 2014, 5, 5824.

15 H. V. Phuc, Superlattices Microstruct., 2015, 88, 518.

16 O. A. C. Nunes, J. Appl. Phys., 2014, 115, 233715.

17 M. Woszczyna, M. Friedemann, K. Pierz, T. Weimann and F. J. Ahlers, J. Appl. Phys., 2011, 110, 351.

18 M. H. T. Dastjerdi, M. Djavid and Z. Mi, Appl. Phys. Lett., 2015, 106, 1166.

19 D. Grimm, C. C. B. Bufon, C. Deneke, P. Atkinson, D. J. Thurmer, F. Schaffel, S. Gorantla, A. Bachmatiuk and O. G. Schmidt, Nano Lett., 2013, 13, 213.

20 C. Vervacke, C. C. B. Bufon, D. J. Thurmer and O. G. Schmidt, RSC Adv., 2014, 4, 9723.

21 X. Liu, J. Zhang, W. Si, L. Xi, B. Eichler, C. Yan and O. G. Schmidt, ACS Nano, 2015, 9, 1158. 
22 Y. Chen, C. Yan and O. G. Schmidt, Adv. Energy Mater., 2013, 3, 1269.

23 I. D. Barcelos, L. G. Moura, R. G. Lacerda and A. Malachias, Nano Lett., 2014, 14, 3919.

24 Q. Wang, E. Wang, Z. Pan, Y. Gao, B. Li, Z. Jia, Y. Wang, X. Li, X. Zhang, X. Ren and Y. Huang, J. Vac. Sci. Technol., B: Nanotechnol. Microelectron.: Mater., Process., Meas., Phenom., 2014, 32, 030603.
25 A. Reina, X. Jia, J. Ho, D. Nezich, H. Son, V. Bulovic, M. S. Dresselhaus and J. Kong, Nano Lett., 2009, 9, 30.

26 A. Reina, H. Son, L. Jiao, B. Fan, M. S. Dresselhaus, Z. F. Liu and J. Kong, J. Phys. Chem. C, 2008, 112, 17741.

27 T. M. G. Mohiuddin, A. Lombardo, R. R. Nair, A. Bonetti, G. Savini, R. Jalil, N. Bonini, D. M. Basko, C. Galiotis, N. Marzari, K. S. Novoselov, A. K. Geim and A. C. Ferrari, Phys. Rev. B: Condens. Matter Mater. Phys., 2009, 79, 205433. 